\title{
End Compactifications and General Compactifications
}

\author{
MATT INSALL \\ PETER A. LOEB \\ MAEGORZATA ANETA MARCINIAK
}

\begin{abstract}
We use the insights of Robinson's nonstandard analysis as a powerful tool to extend and simplify the construction of compactifications of regular spaces. In particular, we deal with the Stone-Čech compactification and compactifications formed from topological ends. For the nonstandard extension of a metric space, the monad of a standard point $x$ is the set of all points infinitesimally close to $x$. Monads of standard points can also be defined for non-metric spaces. The new points of a compactification are formed from equivalence classes of points that are not in the monad of any standard point. Adjoining such points to the original point set allows a better understanding of the relationship between the original space and the set of compactifying points. Our results for end compactifications are established for regular, connected and locally connected spaces. Simple examples of end compactifications are the two point compactification of the real line and the one point compactification of the complex plane. In general, one or more ends form the "far" termination of a non-compact space, while any "hole" in the space corresponds to an end that is "near". Our results on ends extend previous work initiated by Hans Freudenthal on ends understood as equivalence classes of nested sequences of nonempty open sets with compact boundaries. We show, for example, that a product of spaces with at least two non-compact factors has only one end. A brief overview and introduction to nonstandard analysis begins the discussion.
\end{abstract}

2010 Mathematics Subject Classification 03H05, 54D35 (primary); 54J05 (secondary)

Keywords: Compactifications, Stone-Čech compactification, end compactifications, nonstandard methods

\section{Introduction}

We use the insights of Robinson's nonstandard analysis ([12], [10], also see next section) to extend and simplify previous works in the literature on compactifications. In this setting, the "monad" of a standard point $x$ consists of points in the nonstandard 
extension of each standard open neighborhood of $x$; a "remote point" is one not in the monad of any standard paint. A space has at least one remote point if and only if it is not compact. A compactification of a regular space corresponds to an equivalence relation on the set of remote points. The new points of a compactification are formed from equivalence classes of remote points. The resulting compactification is a compact space containing the original point set as a dense subset. The relative topology on that subset is, in general, weaker than the original topology. Compactifications constructed in the literature often employ a continuous map from the original space into a compact space. Our general construction attaching points formed from equivalence classes of remote points allows a better understanding of the relationship between the original space and the set of compactifying points. For example, given a family of bounded real-valued functions on the original space, one can call two remote points equivalent if the nonstandard extension of each of the functions in the family has infinitesimal variation on the two point set. This leads to such compactifications as the Stone-Čech compactification, but constructed here for spaces that need only be regular. Examples of non-compact spaces we will employ in what follows are not new examples of topological spaces; they will, however, serve to illuminate our theory.

We will apply our results to the theory of compactifications formed from topological ends. Well-known examples of end compactifications are the two point compactification of the real line and the one point compactification of the complex plane. The general notion of topological ends was introduced by Freudenthal in [3] to formalizes the intuitive notion of a "hole" in a noncompact space. His work, using sequences, was recently extended by the third author in [11] and then by the first and third author in [7] using nested nets of connected open sets with compact boundaries such that the intersection of their closures is empty. We have eliminated the special assumptions of these previous papers so that our results apply to all regular, connected and locally connected topological spaces. Moreover, the definition presented here extends the notion of ends in those works in the sense that some spaces with intuitive "holes" fail to have ends at the locations of the "holes", but with the definition here they have ends there and at no other locations. ${ }^{1}$ Among our other results is the fact that a product space with two or more noncompact factors has only one end. Again, a simple example is the complex plane.

In other literature, graph-theoretical ends introduced by Halin in [5] use equivalence classes of rays at infinity; those ends are, in general, distinct from Freudenthal's ends. A discussion that compares the two approaches is presented in Diestel and

\footnotetext{
${ }^{1}$ Our results were presented in part at the 2012, Honolulu, Hawaii AMS meeting. The authors are indebted to Tom Cuchta for helpful comments.
} 
Kühn [1]. The use of nonstandard analysis to form general compactifications has had many contributors starting with the work of Abraham Robinson. In [13], Robinson constructs the Stone-Čech compactification of a given topological group or ring, and indeed, he considers arbitrary compactifications of such structures in the same light. In his approach, the kernel, $K$, of a homomorphism defined on the nonstandard extension of the given algebraic structure plays an essential role. As is well-known for universal algebras, such a kernel $K$ gives rise naturally to an equivalence relation, which, in fact, is a congruence on the nonstandard extension of the given structure. Our approach to compactifications of topological spaces, extending work of Salbany and Todorov ([14], [15]), will utilize equivalence relations on the nonstandard extension of a given topological space, independent of any algebraic structure, thereby producing quite general compactifications.

Most recently, nonstandard analysis has been employed by Goldbring [4] to treat graphtheoretical ends. His work does not deal with ends of a topological group, but ends of a topologized graph, namely the Cayley graph of a given finite presentation of the given group. There, the topology is defined by the graph theoretic properties, making the resulting space a proper geodesic space (a metric space in which every closed ball is compact and the distance between any two points is realized by an isometric path from one point to the other). Of course, the graph theoretic properties are derived naturally from properties of the given finitely presented group, but if the group $G$ in question is a topological group to begin with, then the end space of $G$, as considered by Goldbring, is not the same space of "remainders" considered by Robinson. We note that in defining his ends, Goldbring uses path-connectedness, whereas the ends we construct in this work uses the more general notion of topological connectedness. We also do not assume here that our underlying space is locally compact.

\section{Background for Nonstandard Methods}

In this section, we give a very brief introduction to the nonstandard methods used here. The reader is invited to read Loeb and Wolff [10] for an extensive discussion. For simplicity, we start with the complex plane $\mathbb{C}$. Abraham Robinson [12] showed that points can be adjoined to $\mathbb{C}$ to obtain a "space" * $\mathbb{C}$ with the following properties:

(1) Every sequence $s$, function $f$, and relation $r$ extends to an object (denoted by ${ }^{*} s$, ${ }^{*} f$ and ${ }^{*} r$, respectively) that is built from ${ }^{*} \mathbb{C}$ in a way analogous to its original construction using $\mathbb{C}$. 
(2) In particular, elements are adjoined to the set $\mathbb{N}$ of natural numbers. These new elements are greater than every ordinary integer in terms of the extended ordering ${ }^{*} \leq$; the augmented set is the set ${ }^{*} \mathbb{N}$ of nonstandard natural numbers.

(3) When working with ${ }^{*} \mathbb{C}$, the extended objects are called standard objects, but the adjoined points are called nonstandard numbers. Nonstandard numbers, standard objects and members of standard objects are called internal objects. Keisler [8] showed that any object that can be constructed using only internal objects is again internal.

(4) Some objects are introduced that are not members of any standard object; these are called external objects. An object constructed with essential reference to standard objects is external. For example, the set of standard natural numbers is external in $* \mathbb{N}$. Therefore, an internal subset of $* \mathbb{N}$ cannot begin or end precisely at the standard elements of $* \mathbb{N}$.

(5) All statements true for the plane remain true when properly interpreted for ${ }^{*} \mathbb{C}$. The names of objects constructed from $\mathbb{C}$ are replaced with the names of the extension of those objects, and the interpretation must refer only to internal objects. This property is called the transfer principle. If a properly interpreted statement is true for ${ }^{*} \mathbb{C}$, then because it cannot be false, the original statement is true for $\mathbb{C}$. This property is called the downward transfer principle.

(6) Any initial segment of $* \mathbb{N}$ terminating in a standard or nonstandard, ie, infinite natural number is called hyperfinite. Any internal set in internal one-to-one correspondence with such an initial segment of ${ }^{*} \mathbb{N}$ is called hyperfinite. Hyperfinite sets have all of the formal properties of finite sets.

(7) All elements of any set $S$ constructed from $\mathbb{C}$ are members of a hyperfinite subset of ${ }^{*} S$. In general, this principle, with some cardinality conditions, is referred to as saturation.

The metric on $\mathbb{C}$ extends to an internal function taking pairs of points in ${ }^{*} \mathbb{C}$ to the extension of the nonnegative real numbers ${ }^{*} \mathbb{R}^{+}$. The reciprocal of any element from ${ }^{*} \mathbb{R}^{+}$that is larger than all standard natural numbers is a positive infinitesimal number; 0 is the only real infinitesimal. Given a point $p \in \mathbb{C}$, the collection of all points in $* \mathbb{C}$ an infinitesimal distance from $p$ forms the external set $\mu(p)$ called the monad of $p$. The point $p$ is called the standard part of each point in $\mu(p)$. A set $A \subseteq \mathbb{C}$ is open if and only if for each $p \in A, \mu(p) \subseteq{ }^{*} A$. On the other hand, $\mu(p)=\bigcap\left\{{ }^{*} U \mid U\right.$ is open, $p \in U\}$. A similar approach to this treatment of $\mathbb{C}$ and ${ }^{*} \mathbb{C}$ yields nonstandard extensions of metric spaces and also topological spaces, but for topological spaces, the monad of a point $p$ is just defined as the intersection of the extended open neighborhoods 
containing $p$. A subset $S$ of a topological space is compact if and only if every point in ${ }^{*} S$ is nearstandard in ${ }^{*} S$, ie in the monad of a standard point of $S$.

In our work with topological spaces, we will always assume that the nonstandard extension is $\kappa$-saturated, where $\kappa$ is greater than the cardinality of the topology (see Loeb and Wolff [10, Definition 2.9.1]). We will work with what Robinson [12] called the S-topology on such a nonstandard extension. It is the topology generated by the set of nonstandard extensions of standard open sets. Important for our work here is the theorem of Salbany and Todorov (see [14] and [15]) stating that the S-topology is a compact topology on the nonstandard extension, whence the continuous image is compact.

\section{General Compactifications}

Let $(Z, \mathcal{T})$ be a regular noncompact topological space. By regularity, we mean that for each point $p \in Z$, the singleton $\{p\}$ is a closed set and every open neighborhood $U$ of $p$ contains a smaller open neighborhood $V$ of $p$ with closure $\bar{V} \subseteq U$. If there is a compact subset $K_{0}$ of our original space where regularity fails on the interior of $K_{0}$, then we assume that $Z$ consists of the complement of the interior of $K_{0}$. It is well known that compact subsets of $Z$ are closed sets. We now fix a $\kappa$-saturated nonstandard extension of $(Z, \mathcal{T})$, where $\kappa$ is greater than the cardinality of the topology $\mathcal{T}$.

3.1 Definition We call a point $x \in{ }^{*} Z$ remote if $x$ is not nearstandard, ie not in the monad of any standard point of $Z$.

We assume that there is an equivalence relation on the set of remote points of ${ }^{*} Z$. We write $x \sim y$ if $x$ and $y$ are remote and equivalent. The monads of points of $Z$ are equivalence classes in the nearstandard points of ${ }^{*} Z$. In general, the equivalence classes are external.

3.2 Definition Let $Y$ be the point set consisting of points of $Z$, called s-points, together with all equivalence classes of remote points, where each equivalence class is treated as a single point. We call such a point of $Y$ an r-point. We supply ${ }^{*} Z$ with the $S$-topology. Let $\varphi$ be the mapping from ${ }^{*} Z$ onto $Y$ that sends near-standard points to their standard parts and remote points to their respective r-points. The neighborhood filter base $\mathcal{B}(y)$ at an r-point $y$ in $Y$ consists of all sets of the form $\varphi\left({ }^{*} U\right)$, where $U$ is a standard open subset of $Z$ with nonstandard extension containing the entire equivalence class corresponding to $y$ (whence $U \neq \varnothing$ ). The neighborhood filter 
base $\mathcal{B}(x)$ at an s-point $x$ in $Y$ consists of all sets of the form $\varphi\left({ }^{*} U\right)$, where $U$ is a standard open subset of $Z$ with $x \in U$.

3.3 Proposition The neighborhood "filter base" $\mathcal{B}(p)$ at a point $p \in Y$ is in fact a filter base.

Proof Suppose $U$ and $V$ are two standard open subsets of $Z$ such that $\varphi\left({ }^{*} U\right)$ and $\varphi\left({ }^{*} V\right)$ are members of $\mathcal{B}(p)$. Set $W:=U \cap V$. If $p$ is an s-point, then $p \in W$. If $p$ is an r-point, then ${ }^{*} W$ contains the equivalence class corresponding to $p$. Moreover,

$$
\varphi\left({ }^{*} W\right)=\varphi\left({ }^{*}(U \cap V)\right)=\varphi\left({ }^{*} U \cap{ }^{*} V\right) \subseteq \varphi\left({ }^{*} U\right) \cap \varphi\left({ }^{*} V\right) .
$$

As usual, a set $O \subseteq Y$ is called "open" if for each point $p \in O$, there is an element $\varphi\left({ }^{*} U\right) \in \mathcal{B}(p)$ with $\varphi\left({ }^{*} U\right) \subseteq O$. The collection of open sets forms a topology on $Y$. That is, the collection is stable under the operations of taking finite intersections and arbitrary unions. Every nonempty $U \in \mathcal{T}$ is an open neighborhood in $Z$ of its points, so $\varphi\left({ }^{*} U\right)$ is an element of the neighborhood filter base for each of those s-points in $Y$. Note that we have not yet made any claim about the interior with respect to $Y$ of any member of any neighborhood filter base.

3.4 Definition We let $\mathcal{T}_{Y}$ denote the topology on $Y$, ie the collection of open sets generated by the neighborhood filter bases.

3.5 Proposition Let $A$ be a nonempty subset of $Z$. If $x \in Z$ is the standard part of $y \in{ }^{*} A$, then $x=\varphi(y)$ is a point in the $\mathcal{T}$-closure of $A$. If $x \in Z$ is a point in the $\mathcal{T}$-closure of $A$, then $x$ is a point in the $\mathcal{T}_{Y}$-closure of $A$. Moreover, $\varphi\left({ }^{*} A\right)$ is contained in the $\mathcal{T}_{Y}$-closure of the point set $A$.

Proof The first part is well-known and clear. If $x \in Z$ is a point in the $\mathcal{T}$-closure of $A$, then any $\mathcal{T}_{Y}$ open set $W$ that contains $x$ contains a set $\varphi\left({ }^{*} U\right)$ where $U \in \mathcal{T}$ contains $x$. By definition, there is a point $z \in U \cap A$. Since $z \in \varphi\left({ }^{*} U\right) \cap A \subseteq W \cap A$, $x$ is a point in the $\mathcal{T}_{Y}$-closure of $A$. It now follows that any s-point in $\varphi\left({ }^{*} A\right)$ is in the $\mathcal{T}_{Y}$-closure $A$. Suppose $p$ is an r-point in $\varphi\left({ }^{*} A\right)$. Let $W \in \mathcal{T}_{Y}$ contain $p$. By definition, there is a $V \in \mathcal{T}$ with the equivalence class corresponding to $p$ contained in ${ }^{*} V$ and $\varphi\left({ }^{*} V\right) \subseteq W$. Since $p \in \varphi\left({ }^{*} A\right)$, there is a remote point $y \in{ }^{*} A$ such that $y$ is in the equivalence class corresponding to $p$ and $\varphi(y)=p$. By the choice of $V$, $y \in{ }^{*} V$. Since ${ }^{*} V \cap{ }^{*} A \neq \varnothing$, it follows by downward transfer that $V \cap A$ contains 
a point $x$, whence $x$ is in $A$ and also in $\varphi\left({ }^{*} V\right) \subseteq W$. Thus every point in $\varphi\left({ }^{*} A\right)$ is a point of the $\mathcal{T}_{Y}$-closure of $A$.

3.6 Example Let $Z$ be the open unit disc. Let the remote points on the positive realaxis be one equivalence class forming an r-point $p$, and let the remaining remote points form a second equivalence class. Let $U$ be the open disc from which the nonnegative real-axis has been removed. Then $p$ is a point in the $\mathcal{T}_{Y}$-closure of $U$, but $p$ is not an element of $\varphi\left({ }^{*} U\right)$.

3.7 Theorem The map $\varphi$ is a continuous surjection from ${ }^{*} Z$ onto $Y$, whence, $Y$ is compact. Moreover, the point set $Z$ is dense in $Y$ supplied with the $\mathcal{T}_{Y}$-topology. In general, the $\mathcal{T}$-topology on $Z$ is stronger than the relative $\mathcal{T}_{Y}$-topology on $Z$.

Proof Fix $p \in Y$. Also fix $W \in \mathcal{T}_{Y}$ containing $p$, and a $\mathcal{B}(p)$ set $\varphi\left({ }^{*} U\right) \subseteq W$. If $p \in Z$, then $\varphi\left({ }^{*} U\right)$ is the $\varphi$-image of an S-open set ${ }^{*} U$ containing the monad of $p$ in ${ }^{*} Z$. If $p$ is an r-point of $Y$, then $\varphi\left({ }^{*} U\right) \subseteq W$ is the $\varphi$-image of an S-open set ${ }^{*} U$ containing the equivalence class corresponding to $p$. It follows that $\varphi$ is a continuous map from ${ }^{*} Z$ onto $Y$. As noted above, Salbany and Todorov [14] have shown that the S-topology is a compact topology on ${ }^{*} Z$. It follows that the space $\left(Y, \mathcal{T}_{Y}\right)$ is compact. The neighborhood filter base for each point of $Y$ consists of images under $\varphi$ of the nonstandard extensions of nonempty standard open sets, so the point set $Z$ is a dense subset of $Y$. Finally, given $W \in \mathcal{T}_{Y}$, we must show that $W \cap Z \in \mathcal{T}$. Let $x$ be an s-point of $W$. There is a $V \in \mathcal{T}$ with $x \in V$ and $\varphi\left({ }^{*} V\right) \subseteq W$, whence $V \subseteq \varphi\left({ }^{*} V\right) \cap Z \subseteq W \cap Z$; so $W \cap Z \in \mathcal{T}$.

3.8 Definition We say a subset $A$ of ${ }^{*} Z$ is not equivalence class splitting if for each remote point $y \in A$, the equivalence class containing $y$ is entirely contained in $A$.

3.9 Theorem Fix $O \in \mathcal{T}$, and let $A_{O}$ be a set of remote points in ${ }^{*} O$ such that $A_{O}$ is not equivalence class splitting. Assume that $O$ has the following properties with respect to $A_{O}$ : 1) for each $x \in O$ there is a $V_{x} \in \mathcal{T}$ with $x \in V_{x}$ such that the $\mathcal{T}$-closure of $V_{x}$ is contained in $O$ and the remote points in ${ }^{*} V_{x}$ are contained in $A_{O}$. 2) For each $a \in A_{O}$, there is a $V_{a} \in \mathcal{T}$ such that the $\mathcal{T}$-closure of $V_{a}$ is contained in $O$, every remote point in ${ }^{*} V_{a}$ is contained in $A_{O}$, and the equivalence class containing $a$ is contained in ${ }^{*} V_{a}$. Set $W=\bigcup_{x \in O} \varphi\left({ }^{*} V_{x}\right) \cup \bigcup_{a \in A_{O}} \varphi\left({ }^{*} V_{a}\right)$. Then $W \in \mathcal{T}_{Y}$ and $W \cap Z=O$. Moreover, for each $x \in O, x \in W \subseteq \varphi\left({ }^{*} O\right) \in \mathcal{B}(x)$, and for each $p=\varphi(a)$ for $a \in A_{O}, p \in W \subseteq \varphi\left({ }^{*} O\right) \in \mathcal{B}(p)$. If each $O \in \mathcal{T}$ satisfies the above 
assumptions with respect to a corresponding set $A_{O}$, then the $\mathcal{T}$-topology on $Z$ equals the relative $\mathcal{T}_{Y}$-topology on $Z$.

Proof The result follows from the regularity of $\mathcal{T}$ and the definition of openness for $\mathcal{T}_{Y}$.

3.10 Corollary Assume that $O \in \mathcal{T}$ has the property that for each $x \in O$ there is a $V_{x} \in \mathcal{T}$ with $x \in V_{x}$ such that the $\mathcal{T}$-closure of $V_{x}$ is contained in $O$ and ${ }^{*} V_{x}$ is not equivalence class splitting. Set $W=\bigcup_{x \in O} \varphi\left({ }^{*} V_{x}\right)$. Then $W \in \mathcal{T}_{Y}$ and $W \cap Z=O$. If each $O \in \mathcal{T}$ satisfies the above assumption, then the $\mathcal{T}$-topology on $Z$ equals the relative $\mathcal{T}_{Y}$-topology on $Z$.

3.11 Corollary If $\mathcal{T}$ is a locally compact topology on $Z$, then the $\mathcal{T}$-topology on $Z$ equals the relative $\mathcal{T}_{Y}$-topology on $Z$.

Proof For each $O \in \mathcal{T}$, let $A_{O}=\varnothing$, and for each $x \in O$, let $V_{x}$ have compact closure in $O$.

The next result has obvious consequences in terms of local connectivity of the space $\left(Y, \mathcal{T}_{Y}\right)$.

3.12 Corollary If $O \in \mathcal{T}$ is connected and each of the sets $V_{x}, x \in O$, is connected, then $W$ is connected.

Proof It is well-known that any set containing a connected set $S$ and contained in the closure $\bar{S}$ is connected. Therefore, the result follows from Proposition 3.5. One can also use the following Proposition 3.13.

3.13 Proposition The nonstandard extension of a connected open subset of $Z$ is connected in the $S$-topology.

Proof Let $W$ be an open subset of $Z$. Let $\widetilde{U}$ and $\widetilde{V}$ be two nonempty $S$-open subsets of ${ }^{*} W$. There are two collections $\mathcal{U}$ and $\mathcal{V}$ of open subsets of $Z$ such that $\widetilde{U}=\bigcup_{S \in \mathcal{U}}{ }^{*} S$ and $\widetilde{V}=\bigcup_{T \in \mathcal{V}}{ }^{*} T$. Let $U=\bigcup_{S \in \mathcal{U}} S$ and $V=\bigcup_{T \in \mathcal{V}} T$. If there is a point $w \in W \backslash(U \cup V)$, then $w \in{ }^{*} W \backslash(\widetilde{U} \cup \widetilde{V})$, so $\widetilde{U}$ and $\widetilde{V}$ do not form a disconnection of ${ }^{*} W$. If there is a point $w \in U \cap V$, then $w \in \widetilde{U} \cap \widetilde{V}$, so $\widetilde{U}$ and $\widetilde{V}$ do not form a disconnection of ${ }^{*} W$. Therefore, if $\widetilde{U}$ and $\widetilde{V}$ do form a disconnection of ${ }^{*} W$, then $U$ and $V$ form a disconnection of $W$. 
3.14 Theorem Let $\mathcal{Q}$ be a collection of bounded, continuous, real-valued functions on $Z$. Call two remote points $x$ and $y$ of ${ }^{*} Z$ equivalent if for each $f \in \mathcal{Q},{ }^{*} f(x)-{ }^{*} f(y)$ is infinitesimal. Let $Y$ be the compactification for this equivalence relation. For each $r$-point $p$ in $Y$, and each $f \in \mathcal{Q}$, set $\bar{f}(p)$ equal to the standard part of ${ }^{*} f(x)$ for $x$ in the equivalence class corresponding to $p$. Then $\bar{f}$ gives a continuous extension of $f$ from the points of $Z$ to $Y$. Moreover the set of extensions separates the r-points of $Y$.

Proof Let $\bar{f}$ denote the value of the extension of $f \in \mathcal{Q}$ at all points of $Y$. The fact that the set of extensions separates the r-points of $Y$ follows from the definition of the equivalence classes. To establish the continuity of each $\bar{f}$, we fix $a$ and $b$ in $\mathbb{R}$, and show that $W:=\{y \in Y: a<\bar{f}(y)<b\} \in \mathcal{T}_{Y}$. Fix $p \in W$ and $c$ and $d$ in $\mathbb{R}$ with $a<c<\bar{f}(p)<d<b$. Let $U=\{y \in Z: c<f(y)<d\}$. Then $\varphi\left({ }^{*} U\right) \in \mathcal{B}(p)$ and $\varphi\left({ }^{*} U\right) \subseteq W$.

3.15 Remark The last theorem applied to the class of all bounded, continuous, realvalued functions on $Z$ gives an extension of the Stone-Čech compactification construction to regular spaces that may not be completely regular. It is not necessary to imbed $Z$ in a product space to obtain the compactification. It is easy to see that for the application of this construction to $R$. Arens' example of a regular but not completely regular space (see Degundji [2, page 154]), the $\mathcal{T}$-topology is stronger than the relative $\mathcal{T}_{Y}$-topology.

Note that Theorem 3.14 can be extended to a family of functions each taking values in a compact Hausdorff space.

\section{End Compactifications}

Now we assume that $(Z, \mathcal{T})$ is a regular, connected and locally connected, noncompact topological space. If there is a compact subset $K_{0}$ of our original space where regularity or local connectivity fails on the interior of $K_{0}$, then we assume that $Z$ consists of the complement of the interior of $K_{0}$.

It is well known that each component $W$ of the complement of a compact set is an open set since if $x \in W$, then $x$ has a connected open neighborhood that must be entirely contained in $W$. Again, we fix a $\kappa$-saturated nonstandard extension of $(Z, \mathcal{T})$, where $\kappa$ is greater than the cardinality of the topology $\mathcal{T}$.

4.1 Definition Two remote points $x, y$ in ${ }^{*} Z$ are equivalent, and we write $x \sim y$ if there is an internally connected set $A$ containing no nearstandard points with $x \in A$ and $y \in A$. 
Since the union of two connected sets containing a common point is connected, the relation $\sim$ is an equivalence relation in the set of remote points. In general, the equivalence classes are external.

4.2 Definition We call the equivalence class containing a remote point $x \in{ }^{*} Z$ the end of $Z$ represented by $x$. We call the compactification corresponding to the equivalence relation $\sim$ the end compactification of $Z$. We call an open set $O \in \mathcal{T}$ non endsplitting, or simply NES if ${ }^{*} O$ is not equivalence class splitting with respect to the equivalence classes forming ends.

4.3 Example Let $Z$ be the closed square $[0,1] \times[0,1]$ in the $x, y$-plane, but remove the open real interval $(1 / 3,2 / 3)$ from the bottom edge of the square. The topology on $Z$ is the topology inherited from the plane. There is only one end. It consists of all points in the nonstandard extension of the square with positive but infinitesimal $y$-coordinate and $x$-coordinate between $1 / 3$ and $2 / 3$ but not infinitesimally close to either. The standard points $(1 / 3,0)$ and $(2 / 3,0)$ in $Z$ cannot be separated from this end nor from each other by disjoint open neighborhoods in $Y$. The topology $\mathcal{T}$ is strictly stronger than the relative $\mathcal{T}_{Y}$ topology on $Z$. We also note that the net method of Insall and Marciniak [7] does not work to define the end in $Z$.

It is well known that a regular space is locally compact if and only if every compact subset is contained in an open set $W$ with compact closure $\bar{W}$.

4.4 Theorem Let $K$ be a compact subset of $Z$. Assume there is an open set $W \supseteq K$ with compact closure $\bar{W}$. Then all but a finite number of components of $Z \backslash K$ are contained in the compact set $\bar{W}$. It follows that if $Z$ is locally compact, then the complement of any compact subset of $Z$ has only a finite number of components with nonstandard extension containing remote points.

Proof Let $A=\partial \bar{W}$. Then $A$ is a closed subset of $\bar{W}$, and is therefore compact. If $x \in K$, then $W$ is an open neighborhood of $x$ contained in $\bar{W}$, so $x \notin A$. Therefore, $A \subset Z \backslash K$. Cover $A$ with a finite number of connected open sets $S_{j} \subseteq Z \backslash K$. Let $U$ be a component of $Z \backslash K$. If for some $j, S_{j} \cap U \neq \varnothing$, then since $U$ is a component, $S_{j} \subseteq U$. It follows that there are only a finite number of such components $U$. Suppose $U$ is a component of $Z \backslash K$ such that for each $j, S_{j} \cap U=\varnothing$. Also suppose there is a point $p$ that is a point of closure of $U \backslash \bar{W}$. Then $p \notin W$ and $p \notin A$, but $p$ has a connected open neighborhood that intersects $U$, so that neighborhood is contained in $U$, and in particular, $p \in U \backslash \bar{W}$. Therefore, $U \backslash \bar{W}$ is a closed subset of $Z$. It follows 
that $U \backslash \bar{W}=\varnothing$, since $Z$ is connected. It now follows that all but a finite number of components of $Z \backslash K$ are contained in the compact set $\bar{W}$, and thus have nonstandard extensions with no remote points.

Next, we employ an example of a connected and locally connected space that is not locally compact.

4.5 Example Let the point set $Z$ be the countable collection of half open intervals $I_{n}=(0,1]$ indexed by the natural numbers $\mathbb{N}$ together with a single point denoted by 0 . A base for the neighborhood system of each point of each $I_{n}$ is the usual open base inherited from the real line. A typical element of the base for the neighborhood system of 0 is given by a positive $\varepsilon<1$; it is the set

$$
O_{\varepsilon}:=\{0\} \cup \bigcup_{n \in \mathbb{N}}\left\{x \in I_{n}: x<\varepsilon\right\} .
$$

For each $H \in{ }^{*} \mathbb{N} \backslash \mathbb{N}$, the non-infinitesimal points in $I_{H}$ form an end. These are the only ends. The monad of 0 consists of 0 together with the set of infinitesimal points in $I_{n}$ for each $n \in{ }^{*} \mathbb{N}$. Given a standard open neighborhood $U$ of 0 in $Z,{ }^{*} U$ has nonempty intersection with every end. Therefore, to form a member of $\mathcal{B}(0), \varphi\left({ }^{*} U\right)$ must contain the nonstandard interval $I_{H}$ for every unlimited $H$. It follows that the end compactification of $Z$ is not Hausdorff, but a Hausdorff quotient is obtainable by mapping every end to 0 . Note that the number of ends in the compactification depends on the cardinality of $* \mathbb{N}$ in the selected nonstandard extension.

4.6 Proposition Suppose $O \in \mathcal{T}$ and $p \in{ }^{*} \partial O$ is in the monad of a standard point of $Z$. Then $p$ is actually in the monad of a standard point of $\partial O$. It follows that the boundary $\partial O$ of a nonempty open subset $O$ of $Z$ is compact if and only if every point $\alpha \in{ }^{*} \partial O$ is in the monad of a standard point of $Z$.

Proof Suppose $p$ is a point in ${ }^{*} \partial O$ and $p$ is in the monad of a standard point $x \in Z$. Let $U$ be any standard open neighborhood of $x$; then $p \in{ }^{*} U$. Since $p \in{ }^{*} \partial O,{ }^{*} U$ contains points both inside and outside ${ }^{*} O$, so by downward transfer, $U$ contains points both inside and outside $O$. Therefore, $x \in \partial O$, whence $p$ is nearstandard in ${ }^{*} \partial O$. It follows that if all points of $* \partial O$ are in monads of standard points of $Z$, then they are in monads of standard points of $\partial O$, so $\partial O$ is compact. Conversely, if $\partial O$ is compact, then every $\alpha \in{ }^{*} \partial O$ is in the monad of a point of $\partial O \subseteq Z$.

4.7 Proposition If $W$ is a component of the complement of a compact set $K$, then $W$ is non end-splitting.

Journal of Logic \& Analysis 6:7 (2014) 
Proof Let $x$ and $y$ be equivalent remote points, and let $A$ be an internally connected set containing $x$ and $y$ but no nearstandard point. Then $A$ is contained in the complement of ${ }^{*} K$. Moreover, ${ }^{*} W \cup A$ is internally connected, so $A \subseteq{ }^{*} W$.

4.8 Proposition Suppose $Z$ is locally compact. If $x$ and $y$ are non-equivalent remote points in ${ }^{*} Z$, then there is a compact set $K$ such that $x$ and $y$ are in the nonstandard extensions of different components of $Z \backslash K$.

Proof Assume that for every compact set $K, x$ and $y$ are in the nonstandard extension of the same component of $Z \backslash K$. Then by the assumption of local compactness and saturation, there is a nonstandard compact set $C$ containing all near-standard points such that $x$ and $y$ are in the same component of ${ }^{*} Z \backslash C$. It follows that $y \sim x$.

Since $Z$ is connected, the only nonempty subset of $Z$ with empty boundary is $Z$. Clearly, $Z$ is not end-splitting. In general, we have the following result for non-trivial open subsets of $Z$.

4.9 Theorem A nonempty open set $O \varsubsetneqq Z$ has a compact boundary if and only if $O$ is non end-splitting.

Proof Suppose $\partial O$ is compact; then any point of $* \partial O$ is nearstandard in $Z$. Suppose $\alpha \in{ }^{*} O$ is a remote point and $S$ is an internal set containing $\alpha$. If $S$ is internally connected and contains no nearstandard points, then $S \cap{ }^{*} \partial O=\varnothing$. Therefore, since $S$ is internally connected and has nonempty intersection with ${ }^{*} O, S \cap{ }^{*}(Z \backslash \bar{O})=\varnothing$, whence all points of $S$ are in ${ }^{*} O$. Thus the end containing $\alpha$ is contained in ${ }^{*} O$. It follows that $O$ is not end-splitting.

Now suppose $O$ is a standard open set with a boundary that is not compact. It follows from Proposition 4.6 that there is a remote point $\beta$ in ${ }^{*} \partial O$. We will show that $O$ splits the end represented by $\beta$. Since $\beta$ is remote, for each standard point $x \in Z$, there is a standard neighborhood $U$ of $x$ with $\beta \notin^{*} U$. It follows from regularity that there is a smaller standard open set $V_{x}$ with $x \in V_{x} \subset \bar{V}_{x}$ and $\beta \notin{ }^{*} \bar{V}_{x}$. By saturation, there is a hyperfinite collection of internally open sets $V_{j}$ such that the collection contains ${ }^{*} V_{x}$ for each standard point $x \in Z$, and $\beta$ is not in the internal closure of $V_{j}$ for any $j$. The complement of the union of these internal closures contains an internally open and connected neighborhood $U$ of $\beta$. Since $\beta \in{ }^{*} \partial O, U$ contains points both inside and outside ${ }^{*} O$, so $O$ splits the end represented by $\beta$. 
4.10 Corollary Let $\left\{U_{\alpha}\right\}_{\alpha \in \Lambda}$ be a net of nonempty open subsets of $Z$ with compact boundary directed by downward inclusion and having empty intersection of the closures. If $x$ is a remote point in $\bigcap_{\alpha \in \Lambda}{ }^{*} U_{\alpha}$, then all points in the end represented by $x$ are in $\bigcap_{\alpha \in \Lambda}{ }^{*} U_{\alpha}$.

In [7], the first and third authors defined ends of topological spaces in terms of nets of open sets ordered by containment (with $V \geq U$ if $V \subseteq U$ ); the open sets each have nonempty boundary and the collection of open sets has empty intersection of the closures. It is assumed in [7] that for any such net, each member $U$ contains an open subset with compact boundary. The previous corollary and the next result indicate the relationship between the approach in [7] and the approach taken here using nonstandard methods. In particular, when the above assumption is satisfied, the ends obtained in [7] and the ends defined here are in one-to-one correspondence. Example 4.3 is of a space where the assumption of [7] is not satisfied.

4.11 Proposition Let $\left\{U_{\alpha}\right\}_{\alpha \in \Lambda}$ be a net of nonempty connected open subsets of $Z$ with compact boundary directed by downward inclusion and having empty intersection of the closures. Let $x$ and $y$ be remote points in $\bigcap_{\alpha \in \Lambda}{ }^{*} U_{\alpha}$. Then $y$ is in the end represented by $x$, whence this is the same as the end determined by the method of [7] using the net $\left\{U_{\alpha}\right\}_{\alpha \in \Lambda}$.

Proof By saturation, there is an internally connected open set $V$ containing both $x$ and $y$ such that $V \subset \bigcap_{\alpha \in \Lambda}{ }^{*} U_{\alpha}$. If $z$ is a standard point of ${ }^{*} Z$, then for some $U_{\alpha}$, $z \in Z \backslash \bar{U}_{\alpha}$, whence no point of $V$ is in the monad of $z$. Since no point of $V$ is nearstandard, $x$ and $y$ are equivalent.

4.12 Example Let $Z$ be the complex plane from which the rational numbers have been removed from the real interval $(0,1)$. It follows that nonstandard rational numbers strictly between 0 and 1 are not elements of ${ }^{*} Z$. Clearly, $Z$ is not locally compact. As is true for the complex plane with no points removed, nonstandard points outside the extension of every standard bounded set are remote and form a single end. If $\gamma$ is an irrational number between 0 and 1, then points that have not been removed in the monad of $\gamma$ are mapped by $\varphi$ onto $\gamma$. Points in the nonstandard extension of the complex plane in the monad of a removed rational number $q \in(0,1)$ are remote and form a single end. Open discs in $Z$ that are symmetric about the real-axis and intersect that axis in a set with irrational minimum and maximum values are NES. The resulting end compactification is homeomorphic with the extended complex plane. This use of nonstandard methods sharpens the analysis of this example in [7]. 


\section{Product Spaces}

It is well known that the projection of an open set in a product space onto a factor of that space is an open subset of that factor. Fix a product space $\prod_{\alpha \in \mathcal{I}} X_{\alpha}$. Given $p$ in $\prod_{\alpha \in \mathcal{I}} X_{\alpha}$, let $p_{\alpha}$ denote the projection of $p$ onto $X_{\alpha}$. Let st $(\mathcal{I})$ denote the standard indices in ${ }^{*} \mathcal{I}$. For each $\alpha \in \operatorname{st}(\mathcal{I})$, let $\mu_{\alpha}\left(p_{\alpha}\right)$ be the monad of $p_{\alpha}$ in $X_{a}$. The monad of $p$ in the product space is the product

$$
\prod_{\alpha \in \operatorname{st}(\mathcal{I})} \mu_{\alpha}\left(p_{a}\right) \times \prod_{\alpha \in * \mathcal{I} / \operatorname{st}(\mathcal{I})} X_{\alpha}
$$

It follows that a point $p$ in the nonstandard extension of a product is remote if and only if the projection $p_{\alpha}$ is remote for at least one $\alpha \in \operatorname{st}(\mathcal{I})$.

Assume that for every $\alpha \in \mathcal{I}, X_{\alpha}$ is connected and locally connected. Fix an index $\beta \in \mathcal{I}$ and a subset $A$ of $X_{\beta}$. Let $U$ and $V$ be a pair of nonempty open sets in the product space. The projections of $U$ and $V$ on each factor must be nonempty. Assume $U$ and $V$ form a disconnection of $A \times \prod_{\alpha \neq \beta} X_{\alpha}$. Since $U \cup V$ must cover $A \times \prod_{\alpha \neq \beta} X_{\alpha}$, for each $\alpha \neq \beta$ the projections of $U$ and $V$ on the connected set $X_{\alpha}$ have a nonempty intersection. Moreover, the projections form a disconnection of $A$ on $X_{\beta}$. It follows that $A \times \prod_{\alpha \neq \beta} X_{\alpha}$ is connected if and only if $A$ is a connected subset of $X_{\beta}$.

5.1 Theorem Suppose in the above setting $X_{\alpha}$ is compact for each $\alpha \neq \beta$ and $X_{\beta}$ is not compact. Then the number of ends for $\prod_{\alpha \in \mathcal{I}} X_{\alpha}$ is the number of ends for $X_{\beta}$.

Proof The result follows from the fact that a point $p$ in the nonstandard extension of the product is remote if and only if $p_{\beta}$ is remote. Moreover, an internal set in the nonstandard extension of the product is connected and contains no nearstandard points if and only if this is true for its projection onto ${ }^{*} X_{\beta}$.

5.2 Theorem A product space $\prod_{\alpha \in \mathcal{I}} X_{\alpha}$ formed from spaces that are connected and locally connected such that at least two of those spaces are not compact has only one end.

Proof Given a remote point $p_{\beta}$ in ${ }^{*} X_{\beta}$ where $\beta \in \operatorname{st}(\mathcal{I})$, the product $\left\{p_{\beta}\right\} \times^{*} \prod_{\alpha \neq \beta} X_{\alpha}$ is internally connected and contains no nearstandard point. Similarly, given a remote 
point $p_{\gamma}$ in ${ }^{*} X_{\gamma}$ where $\gamma \in \operatorname{st}(\mathcal{I})$ and $\gamma \neq \beta$, the product $\left\{p_{\gamma}\right\} \times{ }^{*} \prod_{\alpha \neq \gamma} X_{\alpha}$ is internally connected and contains no nearstandard point. These two connected sets have points in common, namely those with projection $p_{\beta}$ on ${ }^{*} X_{\beta}$ and projection $p_{\gamma}$ on ${ }^{*} X_{\gamma}$. Therefore, in terms of the equivalence relation for ends, all points of $\left\{p_{\beta}\right\} \times^{*} \prod_{\alpha \neq \beta} X_{\alpha}$ are equivalent to all points of $\left\{p_{\gamma}\right\} \times * \prod_{\alpha \neq \gamma} X_{\alpha}$. Moreover, as noted above, a point is remote in $* \prod_{\alpha \in \mathcal{I}} X_{\alpha}$ if and only if the projection $p_{\beta}$ is remote for at least one $\beta \in \operatorname{st}(\mathcal{I})$.

5.3 Remark Work that remains in our research on end compactifications includes application to box products and to proximity spaces.

\section{References}

[1] R. Diestel and D. Kühn, Graph-theoretical versus topological ends of graphs, Journal of Combinatorial Theory, Series B 87 (2003), 197-206, doi: 10.1016/S00958956(02)00034-5.

[2] J. Dugundji, Topology, Allyn and Bacon, Inc., Boston, 1966.

[3] H. Freudenthal, Über die Enden topologischer Räume und Gruppen,Math. Z33(1931), 692-713, doi: 10.1007/BF01174375.

[4] I. Goldbring, Ends of groups: a nonstandard perspective, Journal of Logic and Analysis 3:7(2011), 1-28, doi: 10.4115/jla.2011.3.7.

[5] R. Halin, Über unendliche Wege in Graphen, Math. Annalen 157(1964), 125-137, doi: 10.1007/BF01362665, 10.1007/BF01362670.

[6] H. Hopf, Enden offener Räume unendliche diskontinuierliche Gruppen, Comment. Math. Helv. 16 (1943/4), 81-100.

[7] M. Insall and M. A. Marciniak, Nets defining ends of topological spaces, Top. Proc. 40 (2012), 1-11.

[8] H. J. Keisler, An infinitesimal approach to stochastic analysis, Mem. Amer. Math. Soc. 48(1984), no 297.

[9] P. A. Loeb, Compactifications of Hausdorff spaces, Proc. Amer. Math. Soc. 22 (1969), 627-634, doi: 10.1090/S0002-9939-1969-0244950-0.

[10] P. A. Loeb and M. Wolff, ed., Nonstandard Analysis for the Working Mathematician, Kluwer Academic Publishers, now Springer, 2000.

[11] M.A. Marciniak, Holomorphic extensions in smooth toric surfaces, Journal of Geometric Analysis, 22 (2012), 911-933, doi: 10.1007/s12220-011-9219-7. 
[12] A. Robinson, Non-standard Analysis, North-Holland, Amsterdam, 1966.

[13] Compactification of groups and rings and nonstandard analysis, Jour. of Symbolic Logic 34(1969), 576-588, doi: 10.2307/2270851.

[14] S. Salbany and T. Todorov, Nonstandard Analysis in Topology: Nonstandard and Standard Compactifications, J. Symbolic Logic, 65(2000), 1836-1840, doi: 10.2307/2695079.

[15] _ Lecture Notes: Nonstandard Analysis in Topology, http://arxiv.org/abs/1107.3323.

Department of Mathematics and Statistics, Missouri University of Science and Technology, 400 W. 12th St., Rolla, MO 65409-0020

Department of Mathematics, University of Illinois,, 1409 West Green St., Urbana, IL 61801

Department of Mathematics, Engineering and Computer Science, LaGuardia Community College, CUNY, 31-10 Thomson Avenue, Long Island City, NY 11101

insall@mst.edu, ploeb@illinois.edu, mmarciniak@lagcc.cuny.edu

Received: 2 August 2014 Revised: 11 October 2014 